\title{
Predictive factors of pathologically node-negative disease for HER2 positive and triple-negative breast cancer after neoadjuvant therapy
}

\author{
Zhiqiang Shi ${ }^{1 \#}$, Xueer Wang ${ }^{2 \#}$, Pengfei Qiu ${ }^{1}$, Yanbing Liu ${ }^{1}$, Tong Zhao ${ }^{1}$, Xiao Sun ${ }^{1}$, Peng Chen ${ }^{1}$, \\ Chunjian Wang ${ }^{1}$, Zhaopeng Zhang ${ }^{1}$, Binbin Cong ${ }^{1}$, Yongsheng Wang ${ }^{1}$
}

${ }^{1}$ Breast Cancer Center, Shandong Cancer Hospital and Institute, Shandong First Medical University and Shandong Academy of Medical Science, Jinan, China; ${ }^{2}$ Department of Radiotherapy, Shandong Cancer Hospital and Institute, Shandong First Medical University and Shandong Academy of Medical Science, Jinan, China

Contributions: (I) Conception and design: Z Shi; (II) Administrative support: Y Wang; (III) Provision of study materials or patients: P Qiu, Y Liu; (IV) Collection and assembly of data: T Zhao, X Sun; (V) Data analysis and interpretation: Z Shi, X Wang; (VI) Manuscript writing: All authors; (VII) Final approval of manuscript: All authors.

"These authors contributed equally to this work.

Correspondence to: Yongsheng Wang. Breast Cancer Center, Shandong Cancer Hospital and Institute, Shandong First Medical University and Shandong Academy of Medical Science, Jinan, China. Email: wangysh2008@aliyun.com.

Background With the improvement of the efficacy of neoadjuvant therapy (NAT) that is guided by molecular subtypes, the rate of pathologically node-negative disease after NAT (ypN0) is increasing for HER2 positive (HER2+) and triple-negative (TN) breast cancer patients. The necessity of axillary surgery for patients with high ypN0 has been questioned. This study aimed to identify patients among HER2+ and TN breast cancer with low risk for axillary metastases after NAT, and, perhaps, they are suitable for selective elimination of axillary surgery staging.

Methods: From January 2010 to August 2018, 865 breast cancer patients who underwent NAT were included in this retrospective clinical study, and 184 patients $(21.3 \%$ 184/865) suffered from TN and HER2+ breast cancer and received full-course NAT. The correlation among clinicopathological characteristics of HER $2+$ and TN breast cancer and ypN0 were analyzed.

Results: Among the 184 HER2 + and TN breast cancer patients, tumor staging, lymph node staging and Ki-67 before NAT, clinically node-negative disease after NAT (ycN0), and breast radiologic and pathologic complete response (bpCR) were correlated with ypN0 $(\mathrm{P}<0.05)$. Lymph node staging before NAT (OR $=0.363, \mathrm{P}<0.001)$, ycN0 $(\mathrm{OR}=4.995, \mathrm{P}<0.001)$ and $\mathrm{bpCR}(\mathrm{OR}=11.285, \mathrm{P}<0.001)$ were the independent effects of ypN0. The ypN0 rate after NAT in cN0/1 patients with bpCR and ycN0 $(97.6 \%, 40 / 41)$ was significantly higher than that in $\mathrm{cN} 2 / 3$ patients $(62.5 \%, 10 / 16)(\mathrm{P}<0.001)$. Among the 37 patients with initial nodal ultrasonography showing cN0 disease, 17 of 17 (100.0\%) with and 18 of 20 (90.0\%) without bpCR had no evidence of residual nodal disease $(\mathrm{P}=0.178)$. Among the 42 patients with cN1 to ycN0, 23 of $24(95.8 \%)$ with and 10 of 18 (55.6\%) without bpCR had no evidence of residual nodal disease $(\mathrm{P}<0.001)$. Patients without bpCR had a relative risk for nodal residual metastases of 10.560 (95\% CI: $2.720-41.003 ; \mathrm{P}<0.001$ ) compared with those with bpCR in $\mathrm{cN} 1$ group.

Conclusions: In terms of HER2 + and TN breast cancer patients, clinical lymph node staging before NAT, ycN0 and bpCR were the independent predictors of ypN0. bpCR was highly correlated with nodal status after NAT. The risk of axillary lymph nodes residual metastases after NAT in the patients of bpCR with cN0 and $\mathrm{cN} 1$ to $\mathrm{ycN} 0$ was less than $5 \%$, thus making it possible to selectively avoid axillary surgery.

Keywords: Neoadjuvant therapy; triple-negative breast cancer; HER2 positive breast cancer; pathologically nodenegative disease 
Submitted Jun 21, 2020. Accepted for publication Nov 27, 2020.

doi: $10.21037 / g s-20-573$

View this article at: http://dx.doi.org/10.21037/gs-20-573

\section{Introduction}

Neoadjuvant therapy (NAT) is the standard therapy for locally advanced breast cancer (LABC) and some stage II to III triple-negative (TN) and HER2 positive (HER2+) breast cancer patients as well. NAT can not only make inoperable breast cancer operability, but also achieve breast-conserving surgery (BCS) for patients who are willing to conserve breast, and play a role in drug sensitivity test in vivo (1). With the improvement of the efficacy of NAT that is guided by the molecular subtypes, pathologic complete response (pCR) increased, from the initial pCR rate of $9-13 \%$ in the era of anthracyclines to $19-26 \%$ with the addition of taxane chemotherapy and as high as $60-70 \%$ with the addition of trastuzumab and pertuzumab in HER2+ disease (1-4). pCR after NAT is considered as a prognostic factor for long-term survival benefit, and it is significantly associated with tumor molecular subtypes (5). HER2 + and TN breast cancer have higher pCR rate than luminal type $(6,7)$. For $70-80 \%$ of patients with TN and HER2+ breast cancer, axillary lymph nodes (ALNs) are negative after NAT (ypN0) (8-10).

From initial LABC to early breast cancer (EBC), NAT indications got better over the past decade (11). NAT allows the opportunity to evaluate the in vivo efficacy of chemotherapy on the primary breast tumor. NAT is now increasingly used in smaller breast tumors (T1/T2) and TN/HER 2 + breast cancer based on this reason, which are linked to the higher pCR (12). In the presence of residual disease after NAT, survival is higher with residual disease in the breast only compared with residual disease in the nodes only and the lowest with residual disease in both. Patients achieving axillary pCR (apCR) have higher 10-year recurrence-free survival and overall survival $(83 \%$ and $85 \%$, respectively) than those with any residual nodal disease (58\% and $55 \%$, respectively) $(13,14)$. Knowing that pCR rate is higher in HER2+ and TN breast cancer compared with luminal type and apCR in these breast cancer patients purporting an improved survival, an important emerging question is whether surgery can be eliminated for patients who obtain apCR after NAT. This study aimed to identify patients among HER2+ and TN breast cancer with low risk for axillary metastases after NAT, and, perhaps, they are suitable for selective elimination of axillary surgery staging.
We presented the following article in accordance with the STROBE reporting checklist (available at http://dx.doi. org/10.21037/gs-20-573).

\section{Methods}

\section{Patients}

We retrospectively included 865 breast cancer patients who underwent NAT in our hospital from January 2010 and August 2018, and 184 patients $(21.3 \%, 184 / 865)$ were TN and HER2+ breast cancer among them. All patients received full-course anthracycline- and/or taxanebased neoadjuvant chemotherapy regimen before surgery. HER2 + patients were given anti-HER2 targeted therapy (trastuzumab) before and after surgery. The specific chemotherapy regimen was displayed as follows: 70 patients used AC/EC-P/T regimen, 81 patients applied AC/EC$\mathrm{PH} / \mathrm{TH}$ regimen, 8 patients adopted TCH regimen, and 25 patients utilized TAC regimen. The study was conducted in accordance with the Declaration of Helsinki (as revised in 2013). The study was approved by Shandong Cancer Hospital Affiliated to Shandong First Medical University Ethics Committee (No. SDTHEC20090223) and individual consent for this retrospective analysis was waived.

\section{Intervention or procedures}

The high expression of Ki-67 was defined as the average ratio of nuclear positive cells to total tumor cells in 10 high power fields, with more than $20 \%$. Before NAT, all patients obtained complete radiologic assessments. Clinically abnormal ALNs got fine needle aspiration (FNA) before NAT. cN0 was defined as no abnormal ALNs that are found in clinical or radiologic examination. In addition, abnormal ALNs that are discovered in clinical or radiologic examination, but FNA negative could still be defined as cNO.

Breast radiologic complete response (brCR) was defined as no residual disease observed in breast on MRI after NAT, and clinically node-negative disease after NAT (ycN0) was defined as no abnormal ALNs in clinical and radiologic examination. Breast pCR (bpCR) referred to no invasive carcinoma in the breast (ypT0/is), and ypN0 marked no 
breast malignant cells in ALNs or sentinel lymph nodes (SLNs). Patients, including those with inflammatory breast cancer, distant metastases, disease progression during NAT, receiving radiotherapy and axillary surgery before surgery, were excluded.

\section{Measures}

Axillary surgical management was based on local surgeons and the ALNs status after NAT. All patients experiencing sentinel lymph node biopsy (SLNB) accepted dual tracer injection of radiolabeled colloid and blue dye. Under ultrasonographic guidance 3-18 h before surgery, ${ }^{99 \mathrm{~m}} \mathrm{Tc}-\mathrm{SC}$ $(1.0-1.2 \mathrm{~mL})$ was injected intra-parenchymally. Blue dye (2-4 mL) was injected subcutaneously around the tumor 10 minutes before surgery.

\section{Statistical analysis}

The data was analyzed with SPSS 26.0 software package. Independent-sample $t$-test was carried out for continuous variables, and Fisher exact test or Pearson $\chi^{2}$ test was conducted for categorical variables. Univariate logistic regression analysis was performed to assess the strength of the correlation between predictive variable and ypN0 after NAT, and a multivariate logistic regression analysis was conducted to identify independent effects of these univariate predictive variables (Take $\mathrm{P}<0.05$ ).

\section{Results}

The median age of these breast cancer patients was 47 [27-70], and the total pCR rate after NAT was $35.3 \%$ $(65 / 184)$. In the choice of breast surgery, $82.6 \%(152 / 184)$ of breast cancer patients received mastectomy, the rest of patients $(17.4 \%, 32 / 184)$ were provided BCS. A total of 25 patients $(13.6 \%)$ received SLNB, 74 patients $(40.2 \%)$ received SLNB and then switched to axillary lymph node dissection (ALND), and 85 patients $(46.2 \%)$ who given ALND directly. The clinicopathologic characteristics of these patients are illustrated in Table 1 .

\section{The correlation between ypNO and clinicopathological characteristics}

The rate of ypN0 in TN and HER2+ breast cancer patients was $57.1 \%(108 / 184)$. A univariate analysis revealed that tumor staging, lymph node staging and Ki-67 before NAT, ycN0, brCR and bpCR after NAT were correlated with ypN0 $(\mathrm{P}<0.05)$, while menopausal status, pathological types and tumor molecular subtypes were not associated with ypN0 ( $>0.05$, Table 1). By multivariate logistic regression analysis, clinical lymph node staging before NAT (OR $=0.363, \mathrm{P}<0.001)$, ycN0 (OR $=4.995, \mathrm{P}<0.001)$ and $\mathrm{bpCR}$ $(\mathrm{OR}=11.285, \mathrm{P}<0.001)$ were the independent effects of ypN0 after NAT $(\mathrm{P}<0.001)$ (Table 2). As for patients with HER 2 + breast cancer, hormone receptor status did not affect ypN0 after NAT $(\mathrm{P}=0.150)$. The univariate analysis of combining clinical lymph node staging before NAT, $\mathrm{ycN} 0$ and bpCR proved that the ypN0 rate after NAT in cN0/1 patients with bpCR and ycN0 $(97.6 \%, 40 / 41)$ was significantly higher than that in $\mathrm{cN} 2 / 3$ patients $(62.5 \%$, 10/16) $(\mathrm{P}<0.001)$.

\section{The correlation between bpCR and pathological ALNs status after NAT in cNO/1 patients}

Among 37 patients with initial nodal ultrasonography showing cN0 disease, the ypN0 and bpCR rates after NAT were $94.6 \%(35 / 37)$ and $45.9 \%(17 / 37)$, respectively. The ypN0 rate in $\mathrm{cN} 0 / 1$ patients with ycN0 but not bpCR and with ycN0 unselected for bpCR were $73.6 \%(28 / 38)$ and $86 \%$ (68/79), respectively. The ypN0 rates of patients with bpCR and non-bpCR after NAT were $100 \%(17 / 17)$ and $90 \%(18 / 20)$, respectively $(\mathrm{P}=0.178)$ (Figure 1$)$.

Among 42 patients with $\mathrm{cN} 1$ to $\mathrm{ycN} 0$, the bpCR and ypN0 rates after NAT were $57.1 \%(24 / 42)$ and $78.6 \%$ (33/42), respectively. The ypN0 rates of patients with bpCR and non-bpCR after NAT were $95.8 \%(23 / 24)$ and $55.6 \%$ $(10 / 18)$, respectively $(\mathrm{P}<0.001)$. Among 40 patients with $\mathrm{cN} 1$ to $\mathrm{ycN} 1$, the bpCR and ypN0 rates after NAT were $20.0 \%(8 / 40)$ and $35.0 \%(14 / 40)$, respectively. The ypN0 rates of patients with bpCR and non-bpCR after NAT were $87.5 \%(7 / 8)$ and $21.9 \%(7 / 32)$, respectively $(\mathrm{P}<0.001)$ (Figure 1). In $32 \mathrm{cN} 1$ patients with bpCR after NAT, only 2 patients were found to have ALNs residual metastasis after NAT: 1 patient had 1 positive ALN, 1 patient had 2 positive ALNs, and no patients had more than 3 positive ALNs on the final pathologic review (Table 3). With the application of relative risk (RR) ratios to compare $\mathrm{cN} 1$ breast cancer patients with and without a bpCR, patients without bpCR had RR for final positive ALNs pathologic findings of 10.560 (95\% CI: $2.720-41.003 ; \mathrm{P}<0.001)$ compared with patients with bpCR. 
Table 1 The correlation between ypN0 after NAT and clinicopathological characteristics

\begin{tabular}{|c|c|c|c|c|}
\hline Characteristics & All patients $(n=184)$ & ypNO (n=105) & $\%$ & $\mathrm{P}$ \\
\hline Menopause & 67 & 35 & 52.2 & \\
\hline No menopause & 117 & 70 & 59.8 & \\
\hline Pathological types & & & & 0.354 \\
\hline Other & 28 & 18 & 64.3 & \\
\hline Ki-67 & & & & 0.004 \\
\hline Low expression & 34 & 13 & 38.2 & \\
\hline High expression & 150 & 92 & 61.3 & \\
\hline $\mathrm{HR}+$ & 52 & 28 & 53.8 & \\
\hline HR- & 37 & 25 & 67.6 & \\
\hline $\mathrm{TN}$ & 95 & 52 & 54.7 & \\
\hline Clinical tumor staging & & & & 0.009 \\
\hline cT1 & 18 & 12 & 66.7 & \\
\hline сT2 & 89 & 56 & 62.9 & \\
\hline cT3 & 34 & 19 & 55.9 & \\
\hline cT4 & 43 & 18 & 41.9 & \\
\hline Radiologic response of the primary tumor on MRI & & & & 0.020 \\
\hline brCR & 72 & 48 & 66.7 & \\
\hline Non-brCR & 48 & 23 & 47.9 & \\
\hline Missing & 64 & 34 & 53.1 & \\
\hline Radiologic response of the lymph nodes & & & & $<0.001$ \\
\hline ycNO & 95 & 77 & 81.1 & \\
\hline Non-ycNO & 89 & 28 & 31.5 & \\
\hline Pathologic response of the primary tumor & & & & $<0.001$ \\
\hline bpCR (ypTo/is) & 79 & 65 & 82.2 & \\
\hline Non-bpCR & 105 & 40 & 38.1 & \\
\hline
\end{tabular}

NAT, neoadjuvant therapy; ypNO, pathologically node-negative disease after NAT; HR, hormone receptor; brCR, breast radiologic complete response; ycN0, clinically node-negative disease after NAT; bpCR, breast pathologic complete response; N, number; HER2+, human epidermal growth factor receptor 2 positive; TN, triple-negative; MRI, magnetic resonance imaging. 
Table 2 Multivariate logistic analysis of ypN0 related factors after NAT

\begin{tabular}{llll}
\hline Characteristics & OR & $95 \% \mathrm{Cl}$ & $\mathrm{P}$ \\
\hline Ki-67 & 1.643 & $0.689-3.866$ & 0.255 \\
Clinical tumor staging & 0.789 & $0.550-1.133$ & 0.200 \\
Clinical lymph node staging & 0.363 & $0.243-0.542$ & $<0.001$ \\
brCR & 1.389 & $0.872-2.212$ & 0.166 \\
ycNo & 4.995 & $2.496-9.993$ & $<0.001$ \\
bpCR & 11.285 & $4.395-28.974$ & $<0.001$ \\
\hline
\end{tabular}

NAT, neoadjuvant therapy; ypN0, pathologically node-negative disease after NAT; brCR, breast radiologic complete response; ycNO, clinically node-negative disease after NAT; bpCR, breast pathologic complete response; OR, odds ratio; Cl, confidence interval.
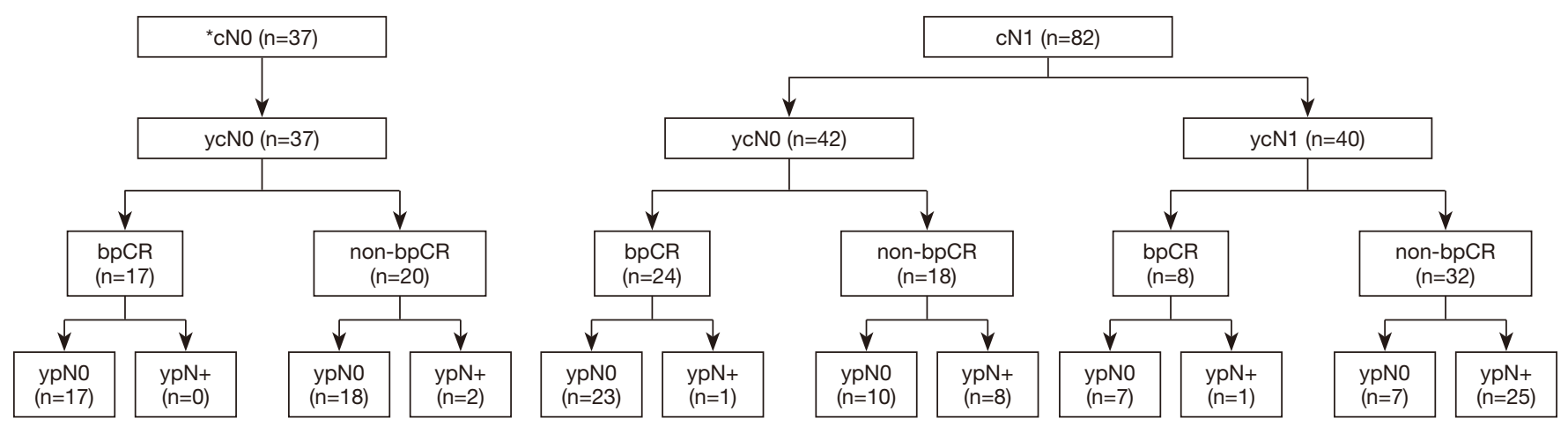

Figure 1 Correlation between bpCR and ypN0 after NAT in cN0/1 patients with HER2+ and TN. NAT, neoadjuvant therapy; bpCR, breast pathologic complete response; ypN0, pathologically node-negative disease after NAT; ypN+, pathologically node-positive disease after NAT; ycN0, clinically node-negative disease after NAT; cN1, initially clinically swollen axillary lymph nodes that can be pushed and metastasis confirmed by fine needle aspiration cytology; ycN1, clinically swollen axillary lymph nodes that can be pushed and metastasis confirmed by fine needle aspiration cytology after NAT. *, cN0 patients with disease progression during NAT were excluded.

Table 3 Pathologic ALNs status in cN1 patients with and without a bpCR after NAT

\begin{tabular}{|c|c|c|c|c|c|c|c|c|}
\hline $\begin{array}{l}\text { Number of positive } \\
\text { ALNs after NAT }\end{array}$ & \multicolumn{3}{|c|}{ Non-bpCR } & \multicolumn{3}{|c|}{ bpCR } & $\mathrm{RR}(95 \% \mathrm{Cl})$ & $\mathrm{P}$ \\
\hline 0 & 9 & 8 & 17 & 15 & 15 & 30 & $10.560(2.720-41.003)$ & $<0.001$ \\
\hline 1 & 6 & 5 & 11 & 0 & 1 & 1 & & \\
\hline 2 & 5 & 4 & 9 & 0 & 1 & 1 & & \\
\hline
\end{tabular}

NAT, neoadjuvant therapy; ALNs, axillary lymph nodes; bpCR, breast pathologic complete response; RR, relative risk; HER2+, human epidermal growth factor receptor 2 positive; TN, triple-negative; $\mathrm{Cl}$, confidence interval. 


\section{Discussion}

From initial LABC to EBC, NAT indications got better over the past decade (11). With the improvement of the efficacy of NAT guided by the molecular subtypes, pCR rate increased, especially in TN and HER $2+$ breast cancer, the pCR rate can reach over $60 \%$ (10). pCR can not only be applied as a prognostic factor for long-term survival of breast cancer patients (5), but also affect the local regional de-escalation management for breast cancer $(15,16)$. NAT not only de-escalates the primary breast tumors to increase the chances of breast-conserving, but also converts positive ALNs patients of approximately $40 \%$ to negative patients (17). In terms of patients with $\mathrm{cN} 1$ to ycN0, $53.6 \%$ of experts believed that, when more than 2 SLNs were detected in the St.Gallen Expert Consensus (in 2017), it could guarantee the accuracy of SLNB after NAT (16). Z1071 test (18) and SENTINA test (19) confirmed that false negative rate (FNR) could be reduced to less than $10 \%$ by using a combined tracer and detecting more than 2 SLNs. NCCN Breast Cancer Clinical Practice Guidelines (in 2017) also recommend SLNB by adopting the above techniques (Class 2B evidence) (15). However, the complications of SLNB were not negligible. Fleissig performed an 18-month randomized controlled trial (14). The results displayed that, although postoperative complications of SLNB were significantly reduced compared with ALND, 7\% of patients had upper extremity edema and $8.7 \%$ complained of arm numbness. Our results exhibited that HER2+ and TN patients with $\mathrm{cN} 0 / 1$ to ycN0 and bpCR had the ypN0 rate of $97.6 \%$ (40/41) after NAT. For this part of patients, whether can we consider avoiding axillary surgery?

At present, the surgical therapy of breast cancer has entered into an era of continuous optimization of deescalation therapy, and even avoiding surgery. The ongoing INSEMA (NCT 02466737) and SOUND studies (NCT 02167490) were designed to explore whether EBC patients with initial nodal ultrasonography showing $\mathrm{cN} 0$ disease could avoid SLNB, while ASICS study was designed to explore the feasibility of avoiding SLNB after NAT in cN0 patients (20). The publication of these three studies may change the current clinical practice, and purport a new thought for cN0 breast cancer, so as to further achieve axillary de-escalation management (avoiding axillary surgery).

MD Anderson Cancer Center (21) conducted a prospective cohort study on 527 HER2 + and TN breast cancer with T1-2N0-1M0 to predict ypN0 by bpCR and carried out a clinical study on avoiding axillary surgery. The results proved that 116 of $116(100.0 \%)$ with and 164 of 174 (94.3\%) without bpCR had no evidence of residual ALNs disease among 290 breast cancer patients with $\mathrm{cN} 0(\mathrm{P}<0.01)$, and 69 of $77(89.6 \%)$ with and 68 of $160(42.5 \%)$ without bpCR had no evidence of residual ALNs disease among 237 breast cancer patients with $\mathrm{cN} 1(\mathrm{P}<0.01)$. Patients without bpCR had RR for ALNs residual metastases of 5.30 (95\% CI: $2.7-10.3, \mathrm{P}<0.001)$ compared with those with bpCR in $\mathrm{cN} 1$ group. Based on the above studies, MD Anderson Cancer Center (22) retrospectively included 30821 patients with T1-2N0-1M0 breast cancer. The results illustrated that the risk of ALNs residual metastases of HER2+ and TN patients with $\mathrm{cN} 0$ after NAT was less than $2 \%$, and axillary surgery could be considered to be avoided. Our results demonstrated that HER2+ and TN patients with cN0/1 to ycN0 and bpCR had ypN0 rate of $97.6 \%(40 / 41)$ after NAT, and the risk of ALNs residual metastases was low, thus making it possible to selectively eliminate axillary surgery staging after NAT, which was consistent with the results of Siso (23). Our results also illustrated that there were 2 cases of ALNs metastases in $\mathrm{cN} 1$ patients with bpCR after NAT, but no pathological findings of more than 3 positive ALNs metastases, suggesting that the residual metastases load of ALNs in cN1 patients with bpCR after NAT was low, and axillary surgery still could be considered to be avoided.

Our research results proved that clinical lymph node staging before NAT, ycN0 and bpCR were the independent predictors of ypN0. Clinical lymph node staging before NAT and ycN0 were easier to be obtained in clinical work, but bpCR could only be learned through surgical pathology, which became the biggest obstacle to predicting ypN0 after NAT. As predictors of bpCR, tumor molecular subtypes, NAT regimens and breast radiologic examination were insufficient in accuracy. However, imaging-guided minimally invasive biopsy (MIB) had the potential to accurately predict bpCR $(24,25)$. Heil (26) performed ultrasound-guided vacuum assisted biopsy (VAB) on 50 patients who achieved brCR after NAT. In histologically representative $V A B$ samples $(n=38)$, the negative predictive value (NPV) and FNR of predicting bpCR were $94.4 \%$ (95\% CI: 87.1-100.0\%) and 4.8\% (95\% CI: 0.0-11.6\%) that were consistent with the results made by MD Anderson Cancer Center study (27). Our central study (data not published, NCT03789851) enrolled 38 patients with radiologic complete and partial response after NAT. 
The NPV and FNR of predicting bpCR by ultrasoundguided multi-point core needle biopsy (CNB) were $84.2 \%$ and $14.3 \%$, respectively. Among them, radioactive iodine seeds were placed around the marker clip before surgery in 26 patients, and then multi-point CNB was performed. The NPV and FNR of predicting bpCR were $92.3 \%$ and $7.7 \%$, respectively. If it can be proved that MIB is safe and efficient when predicting bpCR, patients with bpCR may avoid breast surgery. At the same time, it also overcomes the obstacle that bpCR can only be learned through surgical pathology, thus resulting in the elimination of axillary surgery for the HER2+ and TN patients with $\mathrm{cN} 0 / 1$ to $y c N 0$ and bpCR that are confirmed by MIB. Although patients with occult lymph node metastases may miss the opportunity of additional radiotherapy and/or postneoadjuvant therapies for residual disease for patients who avoid axillary surgery, it can prevent as many patients as possible ( $>95 \%)$ from over-therapy of surgery.

In HER2+ and TN patients, tumor staging, lymph node staging and $\mathrm{Ki}-67$ before NAT, ycN0, brCR and bpCR after NAT were correlated with ypN0. Clinical lymph node staging before NAT, ycN0 and bpCR were the independent predictors of ypN0. bpCR was closely related to ALNs status after NAT. The risk of ALNs residual metastases after NAT in patients with $\mathrm{cN} 0 / 1$ to $\mathrm{ycN} 0$ and bpCR was less than $5 \%$, thereby making it possible to selectively eliminate axillary surgery staging, which would reduce postoperative complications, improve patient quality of life and reduce medical costs. If our research conclusions are extended to clinical work, largescale prospective clinical trials are still needed to study the overall survival rate and local recurrence rate of patients who avoid axillary surgery.

\section{Acknowledgments}

Funding: This work was supported by National Natural Science Foundation of China (81502314) and Medical and Health Science Technology Development Program in Shandong Province (2016WS0549).

\section{Footnote}

Reporting Checklist: The authors have completed the STROBE reporting checklist. Available at http://dx.doi. org/10.21037/gs-20-573

Data Sharing Statement: Available at http://dx.doi. org/10.21037/gs-20-573

Conflicts of Interest: All authors have completed the ICMJE uniform disclosure form (available at http://dx.doi. org/10.21037/gs-20-573). The authors have no conflicts of interest to declare.

Ethical Statement: The authors are accountable for all aspects of the work to ensure that questions that are related to the accuracy or integrity of any part of the work are appropriately investigated and resolved. The study was conducted in accordance with the Declaration of Helsinki (as revised in 2013). The study was approved by Shandong Cancer Hospital Affiliated to Shandong First Medical University Ethics Committee (No. SDTHEC20090223) and individual consent for this retrospective analysis was waived.

Open Access Statement: This is an Open Access article distributed in accordance with the Creative Commons Attribution-NonCommercial-NoDerivs 4.0 International License (CC BY-NC-ND 4.0), which permits the noncommercial replication and distribution of the article with the strict proviso that no changes or edits are made and the original work is properly cited (including links to both the formal publication through the relevant DOI and the license). See: https://creativecommons.org/licenses/by-nc-nd/4.0/.

\section{References}

1. Fisher B, Brown A, Mamounas E, et al. Effect of preoperative chemotherapy on local regional disease in women with operable breast cancer: Findings from National Surgical Adjuvant Breast and Bowel Project B-18. J Clin Oncol 1997;15:2483-93.

2. Bear HD, Anderson S, Smith RE, et al. Sequential preoperative or postoperative docetaxel added to preoperative doxorubicin plus cyclophosphamide for operable breast cancer: National Surgical Adjuvant Breast and Bowel Project Protocol B-27. J Clin Oncol 2006;24:2019-27.

3. Rastogi P, Anderson SJ, Bear HD, et al. Preoperative chemotherapy: updates of National Surgical Adjuvant Breast and Bowel Project Protocols B-18 and B-27. J Clin Oncol 2008;26:778-85.

4. Schneeweiss A, Chia S, Hickish T, et al. Pertuzumab plus trastuzumab in combination with standard neoadjuvant anthracycline-containing and anthracycline-free chemotherapy regimens in patients with HER2-positive 
early breast cancer: a randomized phase II cardiac safety study (TRYPHAENA). Ann Oncol 2013;24:2278-84.

5. Cortazar P, Zhang L, Untch M, et al. Pathological complete response and long term clinical benefit in breast cancer: the CTNeoBC pooled analysis. Lancet 2014;384:164-72.

6. von Minckwitz G, Untch M, Blohmer JU, et al. Definition and impact of pathologic complete response on prognosis after neoadjuvant chemotherapy in various intrinsic breast cancer subtypes. J Clin Oncol 2012;30:1796-1804.

7. Houssami N, Macaskill P, von Minckwitz G, et al. Metaanalysis of the association of breast cancer subtype and pathologic complete response to neoadjuvant chemotherapy. Eur J Cancer 2012;48:3342-54.

8. Boughey JC, McCall LM, Ballman KV, et al. Tumor biology correlates with rates of breast-conserving surgery and pathologic complete response after neoadjuvant chemotherapy for breast cancer: findings from the ACOSOG Z1071 (Alliance) prospective multicenter clinical trial. Ann Surg 2014;260:608-14.

9. Mamtani A, Barrio AV, King TA, et al. How often does neoadjuvant chemotherapy avoid axillary dissection in patients with histologically confirmed nodal metastases? results of a prospective study. Ann Surg Oncol 2016;23:3467-74.

10. Gianni L, Pienkowski T, Im YH, et al. Efficacy and safety of neoadjuvant pertuzumab and trastuzumab in women with locally advanced, inflammatory, or early HER2-positive breast cancer (NeoSphere): a randomised multicentre, open-label, phase 2 trial. Lancet Oncol 2012;13:25-32.

11. Rea D, Tomlins A, Francis A. Time to stop operating on breast cancer patients with pathological complete response? EJSO 2013;39:924-30.

12. Kuerer HM, Newman LA, Smith TL, et al. Clinical course of breast cancer patients with complete pathologic primary tumor and axillary lymph node response to doxorubicin-based neoadjuvant chemotherapy. J Clin Oncol 1999;17:460-9.

13. Mougalian SS, Hernandez M, Lei X, et al. Ten-year outcomes of patients with breast cancer with cytologically confirmed axillary lymph node metastases and pathologic complete response after primary systemic chemotherapy. JAMA Oncol 2016;2:508-16.

14. Fleissig A, Fallowfield LJ, Langridge CI, et al. Postoperative arm morbidity and quality of life. Results of the ALMANAT randomised trial comparing sentinel node biopsy with standard axillary therapy in the management of patients with early breast cancer. Breast Cancer Res Treat 2006;95:279-93.

15. Gradishar WJ, Anderson BO, Balassanian R, et al. Version4.2017, NCCN Clinical Practice Guidelines in Oncology. J Natl Compr Canc Netw 2018;16:310-20.

16. Curigliano G, Burstein HJ, Winer EP, et al. De-escalating and escalating therapys for early-stage breast cancer: the St. Gallen International Expert Consensus Conference on the Primary Therapy of Early Breast Cancer 2017. Ann Oncol 2019;30:1181.

17. Mamounas EP, Brown A, Anderson S, et al. Sentinel node biopsy after neoadjuvant chemotherapy in breast cancer: results from National Surgical Adjuvant Breast and Bowel Project Protocol B-27. J Clin Oncol 2005;23:2694-702.

18. Boughey JC, Suman VJ, Mittendorf EA, et al. Sentinel lymph node surgery after neoadjuvant chemotherapy in patients with node-positive breast cancer: the ACOSOG Z1071(Alliance) clinical trial. JAMA 2013;310:1455-61.

19. Boileau JF, Poirier B, Basik M, et al. Sentinel node biopsy after neoadjuvant chemotherapy in biopsy-proven nodepositive breast cancer: the SNFNAT study. J Clin Oncol 2015;33:258-64.

20. Gentilini O, Veronesi U. Staging the axilla in early breast cancer: will imaging replace surgery? JAMA Oncol 2015;1:1031-2.

21. Tadros AB, Yang WT, Krishnamurthy S, et al. Identification of patients with documented breast pathologic complete response in the breast after neoadjuvant chemotherapy for omission of axillary surgery. JAMA Surg 2017;152:665-70.

22. Barron AU, Hoskin TL, Day CN, et al. Association of Low Nodal Positivity Rate Among Patients With ERBB2-Positive or Triple-Negative Breast Cancer and Breast Pathologic Complete Response to Neoadjuvant Chemotherapy. JAMA Surg 2018;153:1120-6.

23. Siso C, Esgueva A, Rodriguez R, et al. Her2 positive and triple negative breast cancer patients with clinically negative nodes at diagnosis and breast pathologic complete response may spare axillary surgery after neoadjuvant therapy. Eur J Cancer 2018;92:S17-S160.

24. van la Parra RF, Kuerer HM. Selective elimination of breast cancer surgery in exceptional responders: Historical perspective and current trials. Breast Cancer Res 2016;18:28.

25. Kuerer HM, Vrancken Peeters MTFD, Rea DW, et al. Nonoperative Management for Invasive Breast Cancer After Neoadjuvant Systemic Therapy: Conceptual Basis and Fundamental International Feasibility Clinical Trials. 
Ann Surg Oncol 2017;24:2855-62.

26. Heil J, Schaefgen B, Sinn P, et al. Diagnosis of pathological complete response to neoadjuvant chemotherapy in breast cancer by minimal invasive biopsy techniques. Br J Cancer 2015;113:1565-70.

Cite this article as: Shi Z, Wang X, Qiu P, Liu Y, Zhao T, Sun X, Chen P, Wang C, Zhang Z, Cong B, Wang Y. Predictive factors of pathologically node-negative disease for HER2 positive and triple-negative breast cancer after neoadjuvant therapy. Gland Surg 2021;10(1):166-174. doi: 10.21037/gs-20573
27. Kuerer HM, Rauch GM, Krishnamurthy S, et al. A Clinical Feasibility Trial for Identification of Exceptional Responders in Whom Breast Cancer Surgery Can Be Eliminated Following Neoadjuvant Systemic Therapy. Ann Surg 2018;267:946-51. 